\title{
Recommendations for the management of autoinflammatory diseases
}

\author{
Nienke M ter Haar, ${ }^{1,2}$ Marlen Oswald, ${ }^{3}$ Jerold Jeyaratnam, ${ }^{4}$ Jordi Anton, ${ }^{5}$ \\ Karyl S Barron, ${ }^{6}$ Paul A Brogan, ${ }^{7}$ Luca Cantarini, ${ }^{8}$ Caroline Galeotti, ${ }^{9}$ Gilles Grateau, ${ }^{10}$ \\ Veronique Hentgen, ${ }^{11}$ Michael Hofer, ${ }^{12}$ Tilmann Kallinich, ${ }^{13}$ Isabelle Kone-Paut, ${ }^{14}$ \\ Helen J Lachmann, ${ }^{15}$ Huri Ozdogan, ${ }^{16}$ Seza Ozen, ${ }^{17}$ Ricardo Russo, ${ }^{18}$ Anna Simon, ${ }^{19}$ \\ Yosef Uziel, ${ }^{20}$ Carine Wouters, $^{21}$ Brian M Feldman, ${ }^{22}$ Sebastiaan J Vastert, ${ }^{2}$ \\ Nico M Wulffraat, ${ }^{2}$ Susanne M Benseler, ${ }^{23}$ Joost Frenkel, ${ }^{4}$ Marco Gattorno, ${ }^{24}$ \\ Jasmin B Kuemmerle-Deschner ${ }^{3}$
}

\begin{abstract}
Handling editor Tore K Kvien
- Additional material is published online only. To view please visit the journal online (http://dx.doi.org/10.1136/ annrheumdis-2015-207546)

For numbered affiliations see end of article.
\end{abstract}

\section{Correspondence to}

Nienke ter Haar, Department of Paediatric Immunology, and Laboratory for Translational Immunology, University Medical Center Utrecht, Lundlaan 6, Utrecht 3584 EA, The Netherlands;

n.m.terhaar-2@umcutrecht.nl

JF, MG and JBK-D contributed equally.

Received 3 March 2015 Revised 8 June 2015 Accepted 9 June 2015 Published Online First 24 June 2015

\section{ABSTRACT}

Autoinflammatory diseases are characterised by fever and systemic inflammation, with potentially serious complications. Owing to the rarity of these diseases, evidence-based guidelines are lacking. In 2012, the European project Single Hub and Access point for paediatric Rheumatology in Europe (SHARE) was launched to optimise and disseminate regimens for the management of children and young adults with rheumatic diseases, facilitating the clinical practice of paediatricians and (paediatric) rheumatologists. One of the aims of SHARE was to provide evidence-based recommendations for the management of the autoinflammatory diseases cryopyrin-associated periodic syndromes (CAPS), tumour necrosis factor (TNF) receptorassociated periodic syndrome (TRAPS) and mevalonate kinase deficiency (MKD). These recommendations were developed using the European League Against Rheumatism standard operating procedure. An expert committee of paediatric and adult rheumatologists was convened. Recommendations derived from the systematic literature review were evaluated by an online survey and subsequently discussed at a consensus meeting using Nominal Group Technique. Recommendations were accepted if more than $80 \%$ agreement was reached. In total, four overarching principles, 20 recommendations on therapy and 14 recommendations on monitoring were accepted with $\geq 80 \%$ agreement among the experts. Topics included (but were not limited to) validated disease activity scores, therapy and items to assess in monitoring of a patient. By developing these recommendations, we aim to optimise the management of patients with CAPS, TRAPS and MKD.

\section{INTRODUCTION}

Autoinflammatory diseases (AID) are rare disorders that affect multiple organ systems and lead to significant morbidity and mortality. Due to the low patient numbers, evidence-based guidelines for treatment are lacking and management is mostly based on physician's experience. As new effective therapeutic options are now available, early diagnosis and treatment might prevent significant organ damage. Reliable recommendations can thus help paediatricians and (paediatric) rheumatologist in the care of patients with these rare diseases.
In 2012, a European initiative called SHARE (Single Hub and Access point for paediatric Rheumatology in Europe) was launched to optimise and disseminate diagnostic and management regimens in Europe for children and young adults with rheumatic diseases. ${ }^{1}$ One of the aims of SHARE was to provide evidence-based recommendations for the management of paediatric rheumatic diseases.

In this paper, we propose recommendations for the management of three of the main monogenic AID:

1. Cryopyrin-associated periodic syndromes (CAPS), caused by gain-of-function mutations in NLRP3, is a spectrum of diseases that includes the relatively mild familial cold autoinflammatory syndrome (FCAS), the intermediate Muckle-Wells syndrome (MWS) and the severe neonatal-onset multisystem inflammatory disease (NOMID), also known as chronic infantile neurological, cutaneous and articular syndrome (CINCA) and their overlaps (FCAS-MWS and MWS-CINCA). ${ }^{2}$ CAPS is characterised by fever, urticarial rash, conjunctivitis and articular involvement (typically triggered by cold exposure in FCAS); more severely affected patients with CAPS may also have hearing loss, visual loss, chronic meningitis and amyloidosis. $^{3} 4$

2. Tumour necrosis factor (TNF) receptorassociated periodic syndrome (TRAPS) is an autosomal dominant inherited disease. Mutations in TNFRSF1A lead to recurrent fever episodes lasting, on average, 10 days, accompanied by varying symptoms including arthralgia, myalgia and abdominal pain. ${ }^{5}$

3. Mevalonate kinase deficiency (MKD), including hyperimmunoglobulin $\mathrm{D}$ syndrome and the more severe mevalonic aciduria (MA), is an autosomal recessive metabolic inflammatory disease caused by mutations in $M V K$, affecting the mevalonate pathway. ${ }^{6}$ Fever episodes usually last 3-7 days, with lymphadenopathy, abdominal symptoms, arthralgia and a maculopapular skin rash. ${ }^{7}$ In addition to the systemic inflammation, patients with MA have a severe metabolic phenotype, including growth retardation, ataxia and cognitive impairment. $^{8}$ 


\section{METHODS}

An international panel consisting of 22 experts in paediatric or adult rheumatology, internal medicine or nephrology was established to develop evidence-based recommendations for the management and treatment of CAPS, TRAPS and MKD using the European League Against Rheumatism (EULAR) standard operating procedures for developing best practice. ${ }^{9}{ }^{10}$ Eleven of 22 experts were part of the SHARE consortium; 11 additional experts were asked to join the panel based upon their clinical and research expertise in AID.

\section{Systematic literature search}

A systematic literature search was performed in Pubmed, Embase and Cochrane databases on 20 June 2013. All synonyms of CAPS, TRAPS and MKD were searched in MeSH/Emtree terms, title and abstract. Further, we searched on 'autoinflammatory diseases' and synonyms, and manually checked references of relevant original studies and reviews for missing articles. For more details on the literature search, refer to online supplementary figures S1, S2 and S3. Fellows (NMtH, MO, JJ) and experts (JBK-D, SMB, MG, JF) selected the relevant papers for validity assessment. Complete reference lists of full-text screened papers can be found in the online supplement.

\section{Validity assessment}

A panel of experts (two per paper) independently graded the selected papers on methodological quality and extracted data using predefined scoring forms for diagnostic studies, ${ }^{11}$ therapeutic studies ${ }^{12}$ and studies describing prognosis and complications. ${ }^{13}$ Discrepancies were resolved by discussion between the two experts, or by the opinion of a third expert in select instances. Adapted classification tables for diagnostic, ${ }^{14}$ therapeutic $^{9}$ and epidemiological studies ${ }^{15}$ were used to determine the level of evidence and the strength of each recommendation.

\section{Recommendation development}

Recommendations were derived from the available literature and distributed to all experts in an online survey. The drafted recommendations were revised according to comments from the survey and proposed at a face-to-face consensus meeting, where they were discussed by the use of Nominal Group Technique, ${ }^{16}$ supervised by an expert (BMF) in consensus building. Recommendations were accepted when $\geq 80 \%$ of the experts agreed.

\section{RESULTS}

\section{Literature review}

The literature search yielded 1698 unique papers for CAPS, 523 for TRAPS and 618 for MKD. After title/abstract and subsequently full-text screening, 25 CAPS papers, 22 TRAPS papers and $28 \mathrm{MKD}$ papers were assessed for validity and level of evidence, of which $17 / 25,17 / 22$ and $17 / 28$ respectively were considered valid and used for recommendation synthesis. For more details, refer to online supplementary figures S1, S2 and S3.

\section{Recommendations}

In the following section, we describe each recommendation with the corresponding supporting literature and discuss issues regarding practical implementation. Tables 1-3 summarise the recommendations, their levels of evidence, the recommendation strength and percentage of expert agreement for each. Four additional recommendations, specifically on diagnosis of TRAPS and MKD can be found in online supplementary table S1. Recommendations that did not meet $\geq 80 \%$ agreement are listed in online supplementary table S2.

\section{Overarching principles}

AID affect multiple organ systems with potentially severe complications; ${ }^{4} 57817-26$ hence, management of these patients is complex and warrants a multidisciplinary approach: this could involve physiotherapists, paediatricians, rheumatologists and other specialists when specific organs are involved, for example, ENT specialists, ophthalmologists or nephrologists. Furthermore, access to genetic expertise is important because interpretation of genetic results and genetic counselling for family members may be challenging. ${ }^{27}$ For MA, expertise in metabolic diseases is also required.

Given the lifelong duration of AID and impact on patients' and families' life, care should be patient-centred and familycentred, as is the case for most (chronic) diseases. ${ }^{28}$ Patients with CAPS, TRAPS and MKD have an impaired quality of life compared with healthy controls, in both physical and psychosocial domains, and could encounter limitations in education and daily activities. ${ }^{725}$ It is therefore important to offer psychosocial support as appropriate.

Treatment goals should include control of the disease activity and prevention of organ damage, since effective therapy might prevent or stabilise organ damage. ${ }^{21} 3031$ Effective treatment can also improve participation in daily activities and quality of life; this is thus an important additional goal of therapy. ${ }^{31-33}$

\section{Table 1 Overarching principles for CAPS, TRAPS and MKD}

\begin{tabular}{|c|c|c|c|}
\hline Overarching principles & $\mathbf{L}$ & $S$ & Agree (\%) \\
\hline $\begin{array}{l}\text { Management of patients with AID should ideally be guided by a multidisciplinary team in a tertiary centre } \\
\text { with expertise in AID, with access to genetic counselling }\end{array}$ & 4 & D & 100 \\
\hline $\begin{array}{l}\text { The care of patients with AID should include shared patient-centred and family-centred decision-making } \\
\text { with the multidisciplinary team }\end{array}$ & 4 & D & 100 \\
\hline $\begin{array}{l}\text { Aims of the treatment of AID include: } \\
\text { Early and rapid control of disease activity } \\
\text { Prevention of disease and treatment-related damage } \\
\text { Enabling participation in daily activities } \\
\text { Improvement of health-related quality of life }\end{array}$ & 4 & D & 100 \\
\hline In patients with AID, psychosocial support is recommended as appropriate & 4 & D & 100 \\
\hline
\end{tabular}

L, level of evidence; 4, expert opinion; S, strength of recommendation; $D$, based on level 4 evidence. ${ }^{9}$ Agree, percentage of experts who agreed on the recommendation during the final voting round of the consensus meeting.

AID, autoinflammatory diseases; CAPS, cryopyrin-associated periodic syndromes; MKD, mevalonate kinase deficiency; TRAPS, tumour necrosis factor receptor-associated periodic syndrome. 
Table 2 Recommendations for the treatment of CAPS, TRAPS and MKD

$\mathbf{L}$

\section{Treatment CAPS}

IL-1 inhibition is indicated for the whole spectrum of CAPS, at any age

To prevent organ damage, long-term IL-1 inhibition should be started as early as possible in patients with active disease

There is no evidence for the efficacy of DMARDs or biological therapy other than IL-1 blockade in CAPS

For symptomatic adjunctive therapy, short courses of NSAIDs and corticosteroids may be used, ${ }^{\#}$ but they should not be used for primary maintenance therapy ${ }^{\# \#}$

In patients with CAPS, adjunctive therapy (eg, physiotherapy, orthotic devices, hearing aids) is recommended as appropriate

$\begin{array}{lll}1 B-2 A^{*} & A-B & 94.4 \\ 2 B & B & 100 \\ 4 & D & 94.4 \\ { }^{\# 3} 3 & { }^{\# C} C & 100 \\ { }^{\# 4} 4 & { }^{\# \# D} & \\ 4 & D & 100\end{array}$

Treatment TRAPS

NSAIDs may provide symptom relief during inflammatory attacks

Short-term glucocorticoids, with or without NSAIDs, are effective for terminating inflammatory attacks

The beneficial effect of corticosteroids can decline over time so that increasing doses are required to achieve an equivalent response

IL-1 blockade is beneficial in the majority of patients with TRAPS.

Etanercept can be effective in some patients, but the effect might decline over time

With frequent attacks and/or subclinical inflammation between attacks, maintenance therapy with IL-1 blockade or etanercept is

recommended and may limit corticosteroid exposure

If one IL-1 blocking agent at adequate dose is ineffective or intolerable, a switch to etanercept or another IL-1 blocking agent should be considered Likewise, if etanercept is ineffective or intolerable, a switch to an IL-1 blocking agent should be considered

Although a beneficial effect is reported in a few cases, the use of anti-TNF monoclonal antibodies is not advised, due to the possible detrimental effect

Treatment MKD

NSAIDs may provide symptom relief during inflammatory attacks

Short-term glucocorticoids, with or without NSAIDs, may be effective for alleviating inflammatory attacks

Colchicine or statins are not efficacious; therefore we do not recommend their use

Short-term IL-1 blockade may be effective for terminating inflammatory attacks and should be considered to limit or prevent steroid side effects

With frequent attacks and/or subclinical inflammation between attacks, maintenance therapy with IL-1 blockade or etanercept is recommended, and may limit corticosteroid exposure

If one IL-1 blocking agent at adequate dose is ineffective or intolerable, a switch to another IL-1 blocking agent or another biological agent (including TNF- $\alpha$ blockade or IL- 6 blockade) should be considered. Likewise, if TNF- $\alpha$ blockade is ineffective or intolerable, a switch to another biological agent (including an IL-1 or IL-6 blocking agent) should be considered

In selected cases with severe refractory disease with poor quality of life, referral to a specialist centre for consideration of allogeneic haematopoietic stem cell transplantation is recommended.

L, level of evidence; $1 \mathrm{~B}$, randomised controlled study; $2 \mathrm{~A}$, controlled study without randomisation; $2 \mathrm{~B}$, quasi-experimental study; 3 , descriptive study; 4, expert opinion; S, strength of recommendation; $A$, based on level 1 evidence; $B$, based on level 2 or extrapolated from level $1 ; C$, based on level 3 or extrapolated from level 1 or $2 ; D$, based on level 4 or extrapolated from level 3 or 4 evidence. ${ }^{9}$ Agree, percentage of experts who agreed on the recommendation during the final voting round of the consensus meeting.

*See table 4 for detailed information on evidence and approval of IL-1 blocking and TNF-blocking agents.

CAPS, cryopyrin-associated periodic syndromes; DMARDs, disease-modifying antirheumatic drugs; IL, interleukin; MKD, mevalonate kinase deficiency; NSAIDs, non-steroidal anti-inflammatory drugs; TNF, tumour necrosis factor; TRAPS, tumour necrosis factor receptor-associated periodic syndrome.

\section{Diagnosis}

Early diagnosis is crucial to enable treatment initiation before damage occurs. Although diagnostic delay has decreased in the last decades, the median time between onset of symptoms and diagnosis is still 1-2 years. ${ }^{34}$ The diagnostic score for molecular analysis of patients with recurrent fever ${ }^{35}$ and the recently published clinical classification criteria for the diagnosis of monogenic AID $^{36}$ can facilitate the diagnostic process and decrease this delay. We refer to these papers for more details. For CAPS, diagnostic criteria are currently being developed.

Interpretation of genetic tests can also be challenging, especially with low-penetrance TNFRSF1A mutations or NLRP3 mutation-negative CAPS patients. Recently published guidelines for genetic diagnosis of AID can guide physicians and geneticists. ${ }^{27}$ We support the use of these guidelines in the diagnostic process. Specific recommendations on diagnosis, for example, the use of serum immunoglobulin D and urinary mevalonic acid excretion in the diagnostic process of MKD, can be found in supplementary table S1.

\section{Therapy}

\section{Cryopyrin-associated periodic syndromes}

Three interleukin (IL)-1 blocking agents are currently used for CAPS. For a summary of the evidence and the authorisation of the European Medicines Agency (EMA) and Food and Drug Administration (FDA), refer to table 4 and the websites of both organisations. $^{37} 38$

Anakinra was effective in observational studies of patients with CINCA/NOMID (aged 9 months-42 years), 313940 MWS (3-75 years) $^{40-42}$ and adult patients with FCAS. ${ }^{40} 43$ Starting dose of anakinra varied between 0.5 and $2 \mathrm{mg} / \mathrm{kg} /$ day (children) or $100 \mathrm{mg}$ (adults) subcutaneously, but some patients, especially young children, required dose escalation up to 5 or $8 \mathrm{mg} / \mathrm{kg} / \mathrm{day}$ to achieve sustained remission. ${ }^{3142}$ A study on pharmacokinetics of anakinra supports this finding: in order to reach the same effective steady-state concentration, young children need higher doses of anakinra. ${ }^{44}$

Canakinumab was effective in two randomised controlled trials (RCTs) of patients with CAPS (MWS and CINCA/MWS-overlap, aged 9-74 years), ${ }^{32} 45$ and in four observational studies of all disease phenotypes and age categories. ${ }^{40} 424647$ Canakinumab was administered subcutaneously at a dose of $2 \mathrm{mg} / \mathrm{kg}$ (children) or $150 \mathrm{mg}$ (adults) once per 8 weeks, but one study mentions that $24 \%$ of 166 patients required dose escalation up to $8 \mathrm{mg} / \mathrm{kg}$ or $600 \mathrm{mg}$; this was especially the case for paediatric patients or patients with more severe phenotypes. ${ }^{47}$

The efficacy of rilonacept $(2.2 \mathrm{mg} / \mathrm{kg}$ or $160 \mathrm{mg}$ weekly $)$ was demonstrated in one RCT including adult patients with MWS 
L S Agree (\%)

\section{Monitoring - overarching principles}

Monitoring of disease activity and damage is important in patients with AID and should be done regularly

Monitoring frequency should depend on disease severity and activity

The Autoinflammatory Diseases Activity Index (AIDAI) is a validated tool to assess disease activity and should be used in clinical studies of patients with TRAPS and MKD

Physicians should consider other potential causes (eg, infections) when patients experience inflammatory episodes that are atypical of their disease

Prior to therapy with biological agents, consideration should be made to give live and killed vaccines as appropriate. There are currently insufficient safety data to recommend live vaccines during therapy with biological agents.

Monitoring CAPS

Monitoring in all patients with CAPS should include:

- General physical examination, emphasising musculoskeletal and neurological examination, and growth and development of children

- Blood count and inflammatory parameters, such as C-reactive protein (CRP) and serum amyloid A (SAA), if available

- Disease activity, using a validated tool

- Hearing (audiograms) and ophthalmological examination

- Testing for proteinuria

- Impact of disease on well-being, functioning and social participation

For monitoring the disease course of patients with more severe phenotypes, consider including the following tests:

- Cognitive testing

- Lumbar puncture (pressure, cells, protein level)

- Bone MRI and skeletal X-ray

- Brain MRI (including imaging of the inner ear)

Monitoring TRAPS

Monitoring in all patients with TRAPS should include:

- General physical examination and growth and development of children.

- Full blood count and inflammatory parameters, such as CRP and SAA, if available

- Disease activity, using a validated tool

- Testing for proteinuria

- Impact of disease on well-being, functioning and social participation

Interpretation of the significance of the R92Q and P46L sequence variants can be difficult. These occur at a high frequency in healthy controls, and their pathogenic significance remains contentious. Some individuals develop a clinical phenotype of TRAPS, although sometimes with shorter and/or more frequent fever episodes.

Generally, patients carrying R92Q or P46L have milder disease and a better prognosis (improvement over time; and low risk of AA amyloidosis) compared with structural TNFRSF1A mutations.

Patients with chronic, persistent disease activity have a higher risk of developing AA amyloidosis.

Monitoring MKD

Monitoring in all patients with MKD should include:

- General physical examination; and growth and development of children

- Full blood count and inflammatory parameters, such as CRP and SAA, if available

- Disease activity, using a validated tool

- Analysis for proteinuria and haematuria

- Impact of disease on well-being, functioning and social participation

- Ophthalmological examination

For monitoring the disease course of patients with more severe phenotypes, consider including the following examinations:

- Cognitive testing

- Muscle and liver enzymes

- Specific neurological examination

Besides infections, physicians should also be alert to the possibility of macrophage activation syndrome in patients with MKD.

$4 \quad$ D 93.8

$4 \quad$ D 93.8

2B $\quad$ B 100

D 100

D 100

4 D 100

1B $\quad$ B 89.5

2B $\quad$ B 100

4 D 87.5

$4 \quad$ D 100

L, level of evidence; $1 \mathrm{~B}$, prospective cohort study with good follow-up; $2 \mathrm{~B}$, retrospective cohort study, or prospective with poor follow-up; 3, non-consecutive or limited cohort study; 4, case series or expert opinion. S, strength of recommendation; B, based on level 2 or extrapolated from level 1 ; C, based on level 3 or extrapolated from level 1 or 2 ; D, based on level 4 or extrapolated from level 3 or 4 evidence. ${ }^{15}$ Agree, percentage of experts who agreed on the recommendation during the final voting round of the consensus meeting.

AID, autoinflammatory diseases; CAPS, cryopyrin-associated periodic syndromes; MKD, mevalonate kinase deficiency; TRAPS, tumour necrosis factor receptor-associated periodic syndrome.

and FCAS ${ }^{48}$ and two observational studies including patients with MWS and FCAS aged 12-80 years. ${ }^{40} 49$

In CAPS, there is no evidence for the efficacy of diseasemodifying antirheumatic drugs or biological therapy other than IL-1 blockade. ${ }^{50}$ Some patients benefit from non-steroidal antiinflammatory drugs (NSAIDs) and corticosteroids, mostly as on-demand symptom relief next to IL-1 blocking agents. ${ }^{39} 50$ Because of the lack of valid literature on efficacy and prevention of organ damage if used solely, we do not recommend the use of NSAIDs and corticosteroids without IL-1 inhibition.

Organ damage such as hearing loss, neurological damage and joint deformity causes considerable morbidity in patients with CAPS and can partly be stabilised or improved with IL-1 inhibition. $^{21} 303151$ Therefore, it is important to start IL-1 inhibition early, before severe damage occurs. When patients develop irreversible damage, they may need adjunctive therapy such as physiotherapy, orthotic devices and hearing aids.

\section{TNF receptor-associated periodic syndrome}

Evidence for therapy of patients with TRAPS relies on retrospective cohorts or small prospective studies at best. In one retrospective cohort, NSAIDs provided symptom relief in approximately $75 \%$ of patients with TRAPS, but were rarely completely effective in terminating inflammatory episodes. ${ }^{50}$ Efficacy of corticosteroids has only been assessed in 


\section{Recommendation}

Table 4 Summary of evidence and regulatory authorisations for IL-1 blockade and TNF-blockade

\begin{tabular}{|c|c|c|c|}
\hline & $\mathbf{L}$ & EMA approval & FDA approval \\
\hline \multicolumn{4}{|l|}{ CAPS } \\
\hline Canakinumab & 1B & 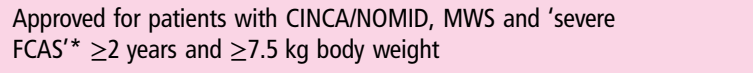 & Approved for patients with FCAS and MWS $\geq 4$ years \\
\hline Rilonacept & 1B & - & Approved for patients with FCAS and MWS $\geq 12$ years \\
\hline Anakinra & $2 \mathrm{~A}$ & Approved for all patients with CAPS $\geq 8$ months and $\geq 10 \mathrm{~kg}$ body weight & Approved for patients with CINCA/NOMID (all ages) \\
\hline \multicolumn{4}{|l|}{ TRAPS } \\
\hline Canakinumab & 3 & Orphan designation & - \\
\hline Anakinra & $2 \mathrm{~B}$ & - & - \\
\hline Etanercept & $2 \mathrm{~B}$ & - & - \\
\hline \multicolumn{4}{|l|}{ MKD } \\
\hline Canakinumab & 3 & - & - \\
\hline Anakinra & $2 B$ & - & - \\
\hline Etanercept & 3 & - & - \\
\hline Adalimumab & 3 & - & - \\
\hline
\end{tabular}

retrospective studies, in which most patients reported a beneficial effect. ${ }^{23} \quad 50 \quad 52-54$ Unfortunately, this initial favourable response often declines with time. ${ }^{53}$

A summary of the evidence and authorisation details for IL-1 blocking and TNF-blocking agents is provided in table 4. Etanercept efficacy was assessed in two small prospective studies; these demonstrated significant improvement in symptoms and inflammatory parameters in most patients but also highlighted declining efficacy over time. This latter finding was the reason for discontinuing etanercept in $11 / 13$ patients in one study. ${ }^{55-57}$ Loss of etanercept efficacy was also reported in larger retrospective studies describing transient responses, and a complete remission rate of less than one-third of patients. ${ }^{50} 58$ Anakinra was effective as on-demand as well as maintenance therapy in a small prospective study. ${ }^{59}$ Retrospective studies confirm this finding; a complete response was reported in two-thirds of the patients. ${ }^{50} 58$ However, anakinra-failure was also observed. ${ }^{5060}$

Because long-term disease activity can cause AA amyloidosis ${ }^{5}$ and long-term use of corticosteroids can induce significant sideeffects, ${ }^{61-63}$ we recommend maintenance therapy with IL-1 blockade or etanercept in patients with frequent attacks and/or ongoing (subclinical) inflammation. In retrospective studies, IL-1 blockade seems to be superior to etanercept. ${ }^{50}$ However, as prospective randomised trials comparing efficacy between these drugs are lacking, insufficient evidence is available to clearly recommend one specific agent as first-line.

Evidence in TRAPS for switching from one biological agent to another is restricted to one study that showed efficacy of anakinra after patients experienced loss of efficacy to etanercept. ${ }^{55}$ Studies in other diseases also suggest that switching could be beneficial and should be considered when the initial treatment choice is ineffective or intolerable. ${ }^{42}{ }^{64}$ For IL-1 blockade, dose adjustments might be necessary to achieve complete response, as has been shown in patients with CAPS. $^{31424447}$

The use of adalimumab and infliximab induces variable results in case reports, including failure and even worsening of TRAPS symptoms; these drugs are thus not recommended. ${ }^{50} 5657$

\section{Mevalonate kinase deficiency}

Treatment efficacy in MKD is mostly described in retrospective cohorts. Most patients benefited from the use of NSAIDs, mainly given during inflammatory attacks. ${ }^{7} 8$ 80 However, NSAIDs are not effective for terminating inflammatory attacks. Similarly, the efficacy of glucocorticoids has only been assessed retrospectively, and most patients derive some therapeutic benefit. 7850 Colchicine and statins are ineffective; moreover, statins induced a severe attack in a patient with MA. ${ }^{7} 82650$

A small prospective study showed that anakinra on demand significantly reduced all features of the fever episode compared with attacks not treated with anakinra. ${ }^{65}$ When patients have frequent attacks and/or subclinical inflammation between attacks, maintenance therapy with IL-1 blockade or etanercept is recommended. Continuous IL-1 blockade with anakinra or canakinumab, or TNF-blockade with etanercept or adalimumab, was beneficial in approximately two-thirds of reported patients. ${ }^{7} 265066$ Because head-to-head comparisons are lacking, it is not possible to recommend any one of these agents as first-line biological therapy. Table 4 summarises the evidence relating to IL- 1 and TNF-blocking agents in MKD. Although there is currently no evidence to support switching biological agents in MKD when initial treatment fails, based on the success of this approach in other diseases, ${ }^{42} 64$ this should be considered. Again, for IL-1 blockade, dose adjustments might be tried first, as the initial dose might be insufficient.

In selected cases with severe refractory disease and poor quality of life, allogeneic haematopoietic stem cell transplantation (HSCT) could be a valuable option. Four cases of successful allogeneic HSCT have been reported: all experienced a remission of systemic inflammation and an improvement of neurological symptoms. ${ }^{67-69}$ Nonetheless, because of the inherent risks associated with HSCT, this should only be considered in severely affected patients resistant to (or intolerant of) all the other aforementioned therapeutic agents.

\section{Monitoring}

General

Since disease-related and treatment-related morbidity is common, and dose adjustment of IL-1 blocking agents to achieve complete 
remission may be required, regular monitoring is crucial. ${ }^{39} 47$ Disease severity varies widely among patients, and thus we recommend that the specific monitoring frequency should be tailored to suit individual patient requirements.

The use of a validated disease activity score is crucial for standardised monitoring of patients with AID. The Autoinflammatory Diseases Activity Index (AIDAI) is the only validated disease activity index for TRAPS and MKD, and one of the validated scores for CAPS. ${ }^{70} 71$ Another validated disease activity score for patients with CAPS is the MWS Disease Activity Score (MWS-DAS). ${ }^{72}$ To increase standardised outcome measurements and thus improve comparisons between studies, we recommend that a validated score should be used in clinical studies of patients with AID: the AIDAI for TRAPS and MKD, and either the AIDAI or MWS-DAS for CAPS.

During monitoring, patients may experience inflammatory episodes that are atypical of their disease; it is important to be aware of other causes of these atypical episodes. Infections are common, especially in children, and might be more common or more dangerous in patients with AID due to their disease and/or immunosuppressive medication. $^{26} 313941-4345474973$ When initiating therapy with biological agents, consideration should be made to give live-attenuated and non-live vaccines as appropriate. According to EULAR recommendations for vaccination in patients with rheumatic diseases, physicians should withhold live-attenuated vaccines when a patient is treated with biological agents. $^{74}$ Some studies suggest that live-attenuated booster vaccination against measles-mumps-rubella and yellow fever can be safely used in patients using TNF-blocking agents. ${ }^{76-78}$ Evidence on safety of vaccinations during IL-1 blocking agents is limited to one randomised study of healthy subjects ${ }^{89}$ and a note that 25 patients with CAPS on canakinumab received vaccinations without abnormal immune responses or pathogenrelated infections. ${ }^{47}$ However, data on live-attenuated vaccines are still scarce; hence we concluded that there are currently insufficient safety data to recommend live vaccines during therapy with biological agents, especially during IL-1 blockade.

\section{Cryopyrin-associated periodic syndromes}

Experts created a list of items that should be included in the monitoring of all patients with CAPS (table 3), based on all relevant symptoms and complications described in patients with CAPS as well as the impact of disease on daily life. $^{17} 18 \quad 2021 \quad 303272 \quad 80-83$ For the more severe CAPS phenotypes, additional tests such as imaging of bone and brain, including inner ear should be considered, as well as cognitive testing and lumbar puncture to assess chronic meningitis. Because bone overgrowth mainly involves the lower limbs, we recommend performing imaging (including skeletal X-ray and MRI) of the femurs, patellae and tibiae.

\section{TNF receptor-associated periodic syndrome}

Most TRAPS symptoms can be assessed by history taking and physical examination. ${ }^{5}$ 22-25 Amyloidosis is described in about $10 \%$ of the patients with TRAPS and occurs more often in patients with long disease duration. ${ }^{522} 2384$ Hence, inflammatory parameters and proteinuria should be checked regularly. ${ }^{5} 22 \quad 23$ A list of particularly relevant items to check when monitoring a patient with TRAPS can be found in table 3 .

Interpretation of the low-penetrance variants R92Q and P46L can be difficult, as $1 \%-2 \%$ of asymptomatic controls also harbour these variants. ${ }^{22} 84$ Patients with R92Q or P46L variants who experience symptoms compatible with TRAPS have a similar phenotype as patients with other TNFRSF1A mutations, although sometimes with shorter and/or more frequent fever episodes. $^{5} 2325$ These patients are also more likely to have a milder disease course (resolution or decrease in fever episodes) and a decreased risk of developing AA amyloidosis compared with patients with structural TNFRSF1A mutations. ${ }^{5} 222325$

\section{Mevalonate kinase deficiency}

Most of the mild MKD symptoms can be assessed by history taking and physical examination and routine laboratory measures such as full blood count, C-reactive protein (CRP) and serum amyloid A (SAA) to assess systemic inflammation. ${ }^{7} 26$ Urine analysis should be performed for renal complications such as AA amyloidosis, glomerulonephritis and renal angiomyolipoma $^{726}$ as well as ophthalmological examination for retinitis pigmentosa and cataracts. ${ }^{2685}$

Patients with more severe MKD phenotypes have neurological complications such as ataxia, hypotonia and psychomotor retardation; thus cognitive testing and full neurological examination should be considered. ${ }^{8} 26$ Since hepatitis and myopathy are also reported in these patients, testing muscle and liver enzymes may be relevant. A list of items particularly relevant for monitoring MKD is provided in table 3.

In addition to an increased infection risk, physicians should also be aware of a higher risk of macrophage activation syndrome (MAS) in patients with MKD, especially in the assessment of atypical fever episodes. MAS is a life-threatening complication of some rheumatic diseases (eg, systemic juvenile idiopathic arthritis), characterised by high fever, pancytopenia and liver damage. ${ }^{86}$ In one cohort of patients with MKD, the frequency of MAS was surprisingly high $(6 / 50,12 \%) .{ }^{26}$

\section{DISCUSSION}

The SHARE taskforce formulated a total of 42 recommendations for the management of patients with the autoinflammatory diseases CAPS, TRAPS and MKD, based on a systematic literature review and consensus procedure.

Biological therapies have dramatically improved the outcome of patients with AID; this is especially true for patients with CAPS on IL-1 blockade. However, evidence supporting the use of biologicals is still very limited in TRAPS and MKD. Prospective studies and head-to-head comparisons are needed to draw solid conclusions on the efficacy of biologicals and to enable EMA/FDA approval. At the time of writing, prospective clinical trials are ongoing: two Phase 2 studies on canakinumab for MKD (NCT01303380) and TRAPS (NCT01242813) and a Phase 3 study on canakinumab for hereditary periodic fevers (NCT02059291). ${ }^{87}$ Regarding the use of IL-1 blockade in very young children with CAPS, the current regulatory approvals by FDA and EMA are provided in table 4. It is recognised, however, that some young patients with severe CAPS may require treatment earlier in life than currently recommended by the authorities. Studies on the safety of canakinumab in the very young are soon-to-be published.

Close monitoring of patients' symptoms, damage and wellbeing is an important requirement for 'treat to target' strategies. Structured long-term follow-up studies of patients with AID are warranted to clarify complication risks. Given the rarity of these diseases, international collaboration is crucial to recruit sufficient patient numbers. Validated scores for disease activity and damage are essential in order to perform a structured assessment of outcome, especially to determine long-term therapeutic efficacy and therapy-associated morbidity. Disease activity scores have now been developed, however, disease damage scores are still lacking. 
A significant limitation of these recommendations is the lack of high-quality evidence. Other than the use of IL-1 blockade in CAPS, most topics are not thoroughly studied. Thus, most of our recommendations have a strength $\mathrm{C}$ or $\mathrm{D}$ and depend mainly on expert opinion. Therefore, further research on complications, monitoring and the use of biological agents in TRAPS and MKD is necessary to enable better evidence-based recommendations. To conclude, based on the best available evidence and expert opinion, this SHARE initiative provides recommendations on diagnosis, treatment and monitoring of CAPS, TRAPS and MKD, aiming to optimise the management of these rare patients.

\section{Author affiliations}

${ }^{1}$ Laboratory for Translational Immunology, University Medical Center Utrecht, Utrecht, Netherlands

${ }^{2}$ Department of Paediatric Immunology, University Medical Center Utrecht, Utrecht, The Netherlands

${ }^{3}$ Division of Paediatric Rheumatology, University Hospital Tuebingen, Tuebingen, Germany

${ }^{4}$ Department of Paediatrics, University Medical Center Utrecht, Utrecht, The Netherlands

${ }^{5}$ Paediatric Rheumatology Unit, Hospital Sant Joan de Déu, University of Barcelona, Barcelona, Spain

${ }^{6}$ Division of Intramural Research, National Institute of Health, Bethesda, USA

${ }^{7}$ Department of Rheumatology, UCL Institute of Child Health, London, UK

${ }^{8}$ Rheumatology Unit, Policlinico Le Scotte, University of Siena, Siena, Italy

${ }^{9}$ Department of Pediatric Rheumatology, Reference Centre for Autoinflammatory

Disorders CEREMAl, Bicêtre Hospital, Paris, France

${ }^{10}$ Department of Internal Medicine, APHP, Hôpital Tenon, University Pierre-et-Marie-

Curie, Paris, France

${ }^{11}$ French Reference Centre for Auto-Inflammatory Diseases in Children, Centre Hospitalier de Versailles, Le Chesnay Cedex, France

${ }^{12}$ Department of Paediatrics, University of Lausanne, Lausanne, Switzerland

${ }^{13}$ Department of Paediatric Pneumology and Immunology, Charité University

Medicine, Berlin, Germany

${ }^{14}$ Department of Paediatric Rheumatology, Reference Centre for Autoinflammatory

Disorders CEREMAI, Bicêtre Hospital, University of Paris SUD, Paris, France

${ }^{15}$ National Amyloidosis Centre, University College London Medical School, London, UK

${ }^{16}$ Division of Rheumatology, Cerrahpasa Ic Hastaliklari Klinigi, University Istanbul, Istanbul, Turkey

${ }^{17}$ Department of Paediatrics, Hacettepe University Faculty of Medicine, Ankara, Turkey

${ }^{18}$ Service of Immunology and Rheumatology, Hospital de Pediatría Garrahan, Buenos Aires, Argentina,

${ }^{19}$ Department of Internal Medicine, Radboud University Medical Center, Nijmegen, The Netherlands

${ }^{20}$ Department of Paediatrics, Meir Medical Center, Tel-Aviv University, Tel-Aviv, Israe

${ }^{21}$ Department of Microbiology and Immunology, University Hospital Leuven, Leuven, Belgium

${ }^{22}$ Division of Rheumatology, The Hospital for Sick Children, Toronto, Canada

${ }^{23}$ Department of Paediatrics, Rheumatology, Alberta Children's Hospital, University

of Calgary, Calgary, Canada

${ }^{24}$ Division of Paediatrics II, G. Gaslini Institute, Genoa, Italy

Acknowledgements This SHARE initiative has been endorsed by the executive committee of the Paediatric Rheumatology European Society (PReS) and the International Society of Systemic Auto-Inflammatory Diseases (ISSAID).

Contributors NMW and SJV designed the SHARE initiative. NMtH, MO and JJ performed the systematic literature review, supervised by JF, JBK-D, SMB and MG. Validity assessment of selected papers was done by MH, IK-P, LC, MG and JBK-D (CAPS), MG, HJL, PAB, GG (TRAPS) and JF, AS, JA, CG (MKD). Recommendations were formulated by $N M H, M O, J F, J B K-D, S M B$ and MG. The expert committee consisted of JA, KSB, PAB, LC, CG, GG, VH, MH, TK, IK-P, HJL, HO, SO, RR, AS, $Y U, C W, S J V, J F, M G$ and JBK-D; they completed the online surveys and/or participated in the subsequent consensus meeting. BMF assisted in the preparation of the live consensus meeting and led the consensus procedure using nominal group technique. NMtH wrote the manuscript, with contribution and approval of all co-authors.

Funding This project is supported by a grant from European Agency for Health and Consumers (EAHC), grant number 20111202.

Competing interests JA: Grant/Research Support from Abbvie, Novartis, Pfizer, Consultant for Novartis, Speaker Bureau of Abbvie, Novartis, Pfizer, Roche, SOBl;
PAB: Grant/Research Support from Novartis, Roche, and SOBI, Consultant for Roche and SOBI; LC: Grant/Research Support from Novartis, SOBI, Consultant for Novartis, SOBI; CG: Grant/Research Support from Novartis; GG: Consultant for Novartis; VH: Consultant for Novartis; MH: Consultant for Novartis; TK: Grant/Research Support from Novartis, Speaker Bureau of Novartis, SOBI; IK-P: Grant/Research Support from Chugai, Novartis, SOBI, Consultant for Abbvie, Chugai, Novartis, Pfizer, SOBI, Speaker Bureau of Novartis, Pfizer; HJL: Research Support and speaker Bureau from Novartis; SO: Consultant for Novartis, Speaker Bureau of SOBI; AS: Consultant for Novartis, Xoma and SOBI; YU: Grant/Research Support from Novartis, Consultant for Novartis, Speaker Bureau of Abbvie, Neopharm, Novartis, Roche; BMF: Consultant for Novartis, Pfizer, BMS; SJV: Consultant for Novartis; NMW: Grant/Research Support from EAHC, Abbvie, GSK, Roche, Consultant for Genzyme, Novartis, Pfizer, Roche; JF: Grant/Research Support from Takeda, Consultant for Novartis, Speaker Bureau of SOBI; MG: Grant/Research Support and speaker Bureau from Novartis and SOBI; JBK-D: Grant/Research Support from Novartis, Speaker Bureau of SOBI.

Provenance and peer review Not commissioned; externally peer reviewed.

\section{REFERENCES}

1 Wulffraat NM, Vastert B, SHARE consortium. Time to share. Pediatr Rheumatol Online J 2013:11:5

2 Aksentijevich I, D Putnam C, Remmers EF, et al. The clinical continuum of cryopyrinopathies: novel CIAS1 mutations in North American patients and a new cryopyrin model. Arthritis Rheum 2007:56:1273-85.

3 Neven B, Prieur AM, Quartier dit Maire P. Cryopyrinopathies: update on pathogenesis and treatment. Nat Clin Pract Rheumatol 2008:4:481-9.

4 Levy R, Gerard L, Kuemmerle-Deschner J, et al. Phenotypic and genotypic characteristics of cryopyrin-associated periodic syndrome: a series of 136 patients from the Eurofever Registry. Ann Rheum Dis 2014. Published Online First: 18 Jul 2014. doi:10.1136/annrheumdis-2013-204991

5 Lachmann HJ, Papa R, Gerhold K, et al. The phenotype of TNF receptor-associated autoinflammatory syndrome (TRAPS) at presentation: a series of 158 cases from the Eurofever/EUROTRAPS international registry. Ann Rheum Dis 2014;73:2160-7.

6 van der Burgh R, Ter Haar NM, Boes ML, et al. Mevalonate kinase deficiency, a metabolic autoinflammatory disease. Clin Immunol 2013;147:197-206.

7 van der Hilst Jeroen $\mathrm{CH}$, Bodar EJ, Barron KS, et al. Long-term follow-up, clinical features, and quality of life in a series of 103 patients with hyperimmunoglobulinemia D syndrome. Medicine 2008;87:301-10.

8 Hoffmann GF, Charpentier C, Mayatepek E, et al. Clinical and biochemical phenotype in 11 patients with mevalonic aciduria. Pediatrics 1993;91(5 I):915-21.

9 Dougados M, Betteridge N, Burmester GR, et al. EULAR standardised operating procedures for the elaboration, evaluation, dissemination, and implementation of recommendations endorsed by the EULAR standing committees. Ann Rheum Dis 2004:63:1172-6.

10 van der Heijde D, Aletaha D, Carmona L, et al. 2014 Update of the EULAR standardised operating procedures for EULAR-endorsed recommendations. Ann Rheum Dis 2015;74:8-13.

11 Whiting P, Rutjes AW, Dinnes J, et al. Development and validation of methods for assessing the quality of diagnostic accuracy studies. Health Technol Assess 2004:8:iii, 1-234.

12 Cochrane Handbook for Systematic Reviews of Interventions Version 5.1.0. The Cochrane Collaboration. http://www.cochrane-handbook.org (accessed Jul 2013).

13 Laupacis A, Wells G, Richardson WS, et al. Users' guides to the medical literature. V. How to use an article about prognosis. Evidence-Based Medicine Working Group. JAMA 1994:272:234-7.

14 Zhang W, Doherty M, Pascual E, et al. EULAR evidence based recommendations for gout. Part I: Diagnosis. Report of a task force of the Standing Committee for International Clinical Studies Including Therapeutics (ESCISIT). Ann Rheum Dis 2006:65:1301-11

15 OCEBM Levels of Evidence Working Group. "The Oxford Levels of Evidence 1." http://www.cebm.net/index.aspx?0=1025 (accessed Jul 2014).

16 Harvey $N$, Holmes CA. Nominal group technique: an effective method for obtaining group consensus. Int I Nurs Pract 2012;18:188-94.

17 Prieur A-M, Griscelli C, Lampert F, et al. A chronic, infantile, neurological, cutaneous and articular (CINCA) syndrome. A specific entity analysed in 30 patients. Scand J Rheumatol Supp/ 1987;66(Suppl 66):57-68.

18 Hoffman HM, Wanderer AA, Broide DH. Familial cold autoinflammatory syndrome: Phenotype and genotype of an autosomal dominant periodic fever. J Allergy Clin Immunol 2001;108:615-20.

19 Arostegui Jl, Aldea A, Modesto C, et al. Clinical and genetic heterogeneity among Spanish patients with recurrent autoinflammatory syndromes associated with the CIAS1/PYPAF1/NALP3 gene. Arthritis Rheum 2004;50:4045-50.

20 Caroli F, Pontillo A, D'Osualdo A, et al. Clinical and genetic characterization of Italian patients affected by CINCA syndrome. Rheumatology 2007:46:473-8.

21 Kuemmerle-Deschner JB, Koitschev A, Ummenhofer K, et al. Hearing loss in Muckle-Wells syndrome. Arthritis Rheum 2013;65:824-31. 
22 Hull KM, Drewe E, Aksentijevich I, et al. The TNF receptor-associated periodic syndrome (TRAPS): Emerging concepts of an autoinflammatory disorder. Medicine 2002;81:349-68.

23 Ravet N, Rouaghe S, Dode C, et al. Clinical significance of P46L and R92Q substitutions in the tumour necrosis factor superfamily $1 \mathrm{~A}$ gene. Ann Rheum Dis 2006;65:1158-62.

24 Lainka E, Neudorf $U$, Lohse $P$, et al. Incidence of TNFRSF1A mutations in German children: epidemiological, clinical and genetic characteristics. Rheumatology 2009;48:987-91.

25 Pelagatti MA, Meini A, Caorsi R, et al. Long-term clinical Profile of children with the low-penetrance R92Q mutation of the tNFRSF1A gene. Arthritis Rheum 2011:63:1141-50.

26 Bader-Meunier B, Florkin B, Sibilia J, et al. Mevalonate kinase deficiency: a survey of 50 patients. Pediatrics 2011;128:e152-9.

27 Shinar Y, Obici L, Aksentijevich I, et al. Guidelines for the genetic diagnosis of hereditary recurrent fevers. Ann Rheum Dis 2012;71:1599-605.

28 Dwamena F, Holmes-Rovner M, Gaulden CM, et al. Interventions for providers to promote a patient-centred approach in clinical consultations. Cochrane Database Syst Rev 2012;12:CD003267.

29 Stych B, Dobrovolny D. Familial cold auto-inflammatory syndrome (FCAS): Characterization of symptomatology and impact on patients' lives. Curr Med Res Opin 2008;24:1577-82.

30 Koitschev A, Gramlich K, Hansmann S, et al. Progressive familial hearing loss in Muckle-Wells syndrome. Acta Otolaryngol 2012;132:756-62.

31 Sibley $\mathrm{CH}$, Plass N, Snow J, et al. Sustained response and prevention of damage progression in patients with neonatal-onset multisystem inflammatory disease treated with anakinra: a cohort study to determine three- and five-year outcomes. Arthritis Rheum 2012;64:2375-86.

32 Kone-Paut I, Lachmann HJ, Kuemmerle-Deschner JB, et al Sustained remission of symptoms and improved health-related quality of life in patients with cryopyrin-associated periodic syndrome treated with canakinumab: Results of a double-blind placebo-controlled randomized withdrawal study. Arthritis Res Ther 2011;13:R202.

33 Lepore L, Paloni G, Caorsi R, et al. Follow-up and quality of life of patients with cryopyrin-associated periodic syndromes treated with Anakinra. J Pediatr 2010;157:310-315.e1.

34 Toplak N, Frenkel J, Ozen S, et al. An international registry on autoinflammatory diseases: the Eurofever experience. Ann Rheum Dis 2012;71:1177-82.

35 Gattorno M, Sormani MP, D'Osualdo A, et al. A diagnostic score for molecular analysis of hereditary autoinflammatory syndromes with periodic fever in children. Arthritis Rheum 2008;58:1823-32.

36 Federici S, Sormani MP, Ozen S, et al. Evidence-based provisional clinical classification criteria for autoinflammatory periodic fevers. Ann Rheum Dis 2015;74:799-805.

37 European public assessment reports. http://www.ema.europa.eu/ema/index.jsp (accessed Jun 2015).

38 FDA Approval labels. http://www.accessdata.fda.gov/scripts/cder/drugsatfda/index. cfm (accessed Jun 2015).

39 Goldbach-Mansky R, Dailey NJ, Canna SW, et al. Neonatal-onset multisystem inflammatory disease responsive to interleukin-1(beta) inhibition. N Engl J Med 2006;355:581-92.

40 Wittkowski H, Kuemmerle-Deschner JB, Austermann J, et al. MRP8 and MRP14, phagocyte-specific danger signals, are sensitive biomarkers of disease activity in cryopyrin-associated periodic syndromes. Ann Rheum Dis 2011;70:2075-81.

41 Kuemmerle-Deschner JB, Tyrrell PN, Koetter I, et al. Efficacy and safety of anakinra therapy in pediatric and adult patients with the autoinflammatory Muckle-Wells syndrome. Arthritis Rheum 2011;63:840-9.


syndrome: Analysis of two IL-1-blocking regimens. Arthritis Res Ther 2013;15:R64.

43 Ross JB, Finlayson LA, Klotz PJ, et al. Use of anakinra (Kineret) in the treatment of familial cold autoinflammatory syndrome with a 16-month follow-up. J Cutan Med Surg 2008;12:8-16.

44 Urien S, Bardin C, Bader-Meunier B, et al. Anakinra pharmacokinetics in children and adolescents with systemic-onset juvenile idiopathic arthritis and autoinflammatory syndromes. BMC Pharmacol Toxicol 2013;14:40.

45 Lachmann HJ, Kone-Paut I, Kuemmerle-Deschner JB, et al. Use of canakinumab in the cryopyrin-associated periodic syndrome. N Engl J Med 2009;360:2416-25.

46 Kuemmerle-Deschner JB, Ramos E, Blank N, et al. Canakinumab (ACZ885, a fully human IgG1 anti-IL-1beta mAb) induces sustained remission in pediatric patients with cryopyrin-associated periodic syndrome (CAPS). Arthritis Res Ther 2011;13:R34.

47 Kuemmerle-Deschner JB, Hachulla E, Cartwright R, et al. Two-year results from an open-label, multicentre, phase III study evaluating the safety and efficacy of canakinumab in patients with cryopyrin-associated periodic syndrome across different severity phenotypes. Ann Rheum Dis 2011;70:2095-102.

48 Hoffman HM, Throne ML, Amar NJ, et al. Efficacy and safety of rilonacept (Interleukin-1 Trap) in patients with cryopyrin-associated periodic syndromes: results from two sequential placebo-controlled studies. Arthritis Rheum 2008;58:2443-52.
49 Hoffman HM, Throne ML, Amar NJ, et al. Long-Term Efficacy and Safety Profile of Rilonacept in the Treatment of Cryopryin-Associated Periodic Syndromes: results of a 72-Week Open-Label Extension Study. Clin Ther 2012;34:2091-103.

50 ter Haar NM, Lachmann $\mathrm{H}$, Ozen $\mathrm{S}$, et al. Treatment of autoinflammatory diseases: results from the Eurofever Registry and a literature review. Ann Rheum Dis 2013;72 (5):678-85

51 Neven B, Marvillet I, Terrada C, et al. Long-term efficacy of the interleukin-1 receptor antagonist anakinra in ten patients with neonatal-onset multisystem inflammatory disease/chronic infantile neurologic, cutaneous, articular syndrome. Arthritis Rheum 2010;62:258-67.

52 Williamson LM, Hull D, Mehta R. Familial hibernian fever. Q J Med 1982;51:469-80

53 McDermott EM, Smillie DM, Powell RJ, et al. Clinical spectrum of familial Hibernian fever: a 14-year follow-up study of the index case and extended family. Mayo Clin Proc 1997;72:806-17.

54 Stojanov S, Dejaco C, Lohse P, et al. Clinical and functional characterisation of a novel TNFRSF1A c.605T>A/V173D cleavage site mutation associated with tumour necrosis factor receptor-associated periodic fever syndrome (TRAPS), cardiovascular complications and excellent response to etanercept. Ann Rheum Dis 2008;67:1292-8

55 Bulua AC, Mogul DB, Aksentijevich I, et al. Efficacy of etanercept in the tumor necrosis factor receptor-associated periodic syndrome: a prospective, open-label, dose-escalation study. Arthritis Rheum 2012;64:908-13.

56 Drewe E, McDermott EM, Powell PT, et al. Prospective study of anti-tumour necrosis factor receptor superfamily $1 \mathrm{~B}$ fusion protein, and case study of anti-tumour necrosis factor receptor superfamily $1 \mathrm{~A}$ fusion protein, in tumour necrosis factor receptor associated periodic syndrome (TRAPS): Clinical and laboratory findings in a series of seven patients. Rheumatology 2003;42:235-9.

57 Drewe E, Powell RJ, Mcdermott EM. Comment on: Failure of anti-TNF therapy in TNF Receptor 1-Associated Periodic Syndrome (TRAPS). Rheumatology 2007:46:1865-6

58 Lane T, Loeffler JM, Rowczenio DM, et al. AA amyloidosis complicating the hereditary periodic fever syndromes. Arthritis Rheum 2013;65:1116-21.

59 Gattorno M, Pelagatti MA, Meini A, et al. Persistent efficacy of anakinra in patients with tumor necrosis factor receptor-associated periodic syndrome. Arthritis Rheum 2008:58:1516-20.

60 Quillinan N, Mannion G, Mohammad A, et al. Failure of sustained response to etanercept and refractoriness to anakinra in patients with T50M TNF-receptor-associated periodic syndrome. Ann Rheum Dis 2011;70:1692-3.

61 Hoes JN, Jacobs JW, Boers M, et al. EULAR evidence-based recommendations on the management of systemic glucocorticoid therapy in rheumatic diseases. Ann Rheum Dis 2007;66:1560-7.

62 van der Goes MC, Jacobs JW, Boers M, et al. Monitoring adverse events of low-dose glucocorticoid therapy: EULAR recommendations for clinical trials and daily practice. Ann Rheum Dis 2010;69:1913-9.

63 Duru N, van der Goes MC, Jacobs JW, et al. EULAR evidence-based and consensus-based recommendations on the management of medium to high-dose glucocorticoid therapy in rheumatic diseases. Ann Rheum Dis 2013;72:1905-13.

64 Smolen JS, Landewe R, Breedveld FC, et al. EULAR recommendations for the management of rheumatoid arthritis with synthetic and biological disease-modifying antirheumatic drugs: 2013 update. Ann Rheum Dis 2014;73:492-509.

65 Bodar EJ, Kuijk LM, Drenth JPH, et al. On-demand anakinra treatment is effective in mevalonate kinase deficiency. Ann Rheum Dis 2011;70:2155-8.

66 Galeotti C, Meinzer U, Quartier $P$, et al. Efficacy of interleukin-1-targeting drugs in mevalonate kinase deficiency. Rheumatology (United Kingdom) 2012;51(14620332 (Electronic)):1855-9.

67 Arkwright PD, Abinun M, Cant AJ. Mevalonic aciduria cured by bone marrow transplantation. N Engl I Med 2007;357:1350.

68 Neven B, Valayannopoulos V, Quartier P, et al. Allogeneic bone marrow transplantation in mevalonic aciduria. N Engl J Med 2007;356:2700-3.

69 Chaudhury S, Hormaza L, Mohammad S, et al. Liver transplantation followed by allogeneic hematopoietic stem cell transplantation for atypical mevalonic aciduria. Am J Transplant 2012;12:1627-31.

70 Piram M, Frenkel J, Gattorno M, et al. A preliminary score for the assessment of disease activity in hereditary recurrent fevers: Results from the AIDAI (Auto-Inflammatory Diseases Activity Index) consensus conference. Ann Rheum Dis 2011;70:309-14.

71 Piram M, Koné-Paut I, Lachmann HJ, et al. Validation of the Auto-Inflammatory Diseases Activity Index (AIDAI) for hereditary recurrent fever syndromes. Ann Rheum Dis 2014;73:1-6.

72 Kummerle-Deschner JB, Tyrrell PN, Reess F, et al. Risk factors for severe Muckle-Wells syndrome. Arthritis Rheum 2010;62:3783-91.

73 Singh JA, Wells GA, Christensen R, et al. Adverse effects of biologics: a network meta-analysis and Cochrane overview. Cochrane Database Syst Rev 2011;(2): CD008794.

74 Heijstek MW, Ott de Bruin LM, Bijl M, et al. EULAR recommendations for vaccination in paediatric patients with rheumatic diseases. Ann Rheum Dis 2011;70:1704-12. 


\section{Recommendation}

75 van Assen S, Agmon-Levin N, Elkayam O, et al. EULAR recommendations for vaccination in adult patients with autoimmune inflammatory rheumatic diseases. Ann Rheum Dis 2011;70:414-22.

76 Heijstek MW, Kamphuis S, Armbrust W, et al Effects of the live attenuated measles-mumps-rubella booster vaccination on disease activity in patients with juvenile idiopathic arthritis: a randomized trial. JAMA 2013;309:2449-56.

77 Borte S, Liebert UG, Borte M, et al. Efficacy of measles, mumps and rubella revaccination in children with juvenile idiopathic arthritis treated with methotrexate and etanercept. Rheumatology (Oxford) 2009;48:144-8.

78 Scheinberg M, Guedes-Barbosa LS, Mangueira C, et al. Yellow fever revaccination during infliximab therapy. Arthritis Care Res (Hoboken) 2010;62:896-8.

79 Chioato A, Noseda E, Felix SD, et al. Influenza and meningococcal vaccinations are effective in healthy subjects treated with the interleukin-1 beta-blocking antibody canakinumab: results of an open-label, parallel group, randomized, single-center study. Clin Vaccine Immunol 2010;17:1952-7.

80 Dollfus $\mathrm{H}$, Hafner $\mathrm{R}$, Hofmann $\mathrm{HM}$, et al. Chronic infantile neurological cutaneous and articular/neonatal onset multisystem inflammatory disease syndrome: Ocular manifestations in a recently recognized chronic inflammatory disease of childhood. Arch Ophthalmol 2000;118(0003-9950 (Print)):1386-92.
81 Hill SC, Namde M, Dwyer A, et al. Arthropathy of neonatal onset multisystem inflammatory disease (NOMID/CINCA). Pediatr Radiol 2007;37:145-52.

82 Lainka E, Neudorf U, Lohse P, et al. Analysis of Cryopyrin-associated periodic syndromes (CAPS) in German children: Epidemiological, clinical and genetic characteristics. Klin Padiatr 2010;222:356-61.

83 Rowczenio DM, Trojer H, Russell T, et al. Clinical characteristics in subjects with NLRP3 V198M diagnosed at a single UK center and a review of the literature. Arthritis Res Ther 2013:15:R30.

84 Aksentijevich I, Galon J, Soares M, et al. The tumor-necrosis-factor receptor-associated periodic syndrome: new mutations in TNFRSF1A, ancestral origins, genotype-phenotype studies, and evidence for further genetic heterogeneity of periodic fevers. Am I Hum Genet 2001;69:301-14.

85 Siemiatkowska AM, van den Born LI, van Hagen PM, et al. Mutations in the mevalonate kinase (MVK) gene cause nonsyndromic retinitis pigmentosa. Ophthalmology 2013;120:2697-705.

86 Minoia F, Davi S, Horne A, et al. Clinical features, treatment, and outcome of macrophage activation syndrome complicating systemic juvenile idiopathic arthritis: a multinational, multicenter study of 362 patients. Arthritis Rheumatol 2014;66:3160-9.

87 Clinical Trials Registry. https://clinicaltrials.gov (accessed Feb 2015). 


\section{ARD Recommendations for the management of autoinflammatory diseases}

Nienke M ter Haar, Marlen Oswald, Jerold Jeyaratnam, Jordi Anton, Karyl S Barron, Paul A Brogan, Luca Cantarini, Caroline Galeotti, Gilles Grateau, Veronique Hentgen, Michael Hofer, Tilmann Kallinich, Isabelle Kone-Paut, Helen J Lachmann, Huri Ozdogan, Seza Ozen, Ricardo Russo, Anna Simon, Yosef Uziel, Carine Wouters, Brian M Feldman, Sebastiaan J Vastert, Nico M Wulffraat, Susanne M Benseler, Joost Frenkel, Marco Gattorno and Jasmin B Kuemmerle-Deschner

Ann Rheum Dis 2015 74: 1636-1644 originally published online June 24, 2015

doi: 10.1136/annrheumdis-2015-207546

Updated information and services can be found at:

http://ard.bmj.com/content/74/9/1636

These include:

Supplementary Supplementary material can be found at:

Material http://ard.bmj.com/content/suppl/2015/06/24/annrheumdis-2015-2075 46.DC1

References This article cites 79 articles, 31 of which you can access for free at: http://ard.bmj.com/content/74/9/1636\#BIBL

Email alerting Receive free email alerts when new articles cite this article. Sign up in the service box at the top right corner of the online article.

Topic Articles on similar topics can be found in the following collections

Collections

Connective tissue disease (4253)

Musculoskeletal syndromes (4951)

Guidelines (27)

Immunology (including allergy) (5144)

Inflammation (1251)

\section{Notes}

To request permissions go to:

http://group.bmj.com/group/rights-licensing/permissions

To order reprints go to:

http://journals.bmj.com/cgi/reprintform

To subscribe to BMJ go to:

http://group.bmj.com/subscribe/ 